\title{
Melting heat transfer in steady laminar flow over a moving surface.
}

\begin{abstract}
The steady laminar boundary layer flow and heat transfer from a warm, laminar liquid flow to a melting surface moving parallel to a constant free stream is studied in this paper. The continuity, momentum and energy equations, which are coupled nonlinear partial differential equations are reduced to a set of two nonlinear ordinary differential equations, before being solved numerically using the Runge-Kutta-Fehlberg method.Results for the skin friction coefficient, local Nusselt number, velocity profiles as well as temperature profiles are presented for different values of the governing parameters. Effects of the melting parameter, moving parameter and Prandtl number on the flow and heat transfer characteristics are thoroughly examined. It is found that the problem admits dual solutions.
\end{abstract}

Keyword: melting; Moving surface; Heat transfer. 